\title{
Study protocol of the LARK (TROG 17.03) clinical trial: a phase II trial investigating the dosimetric impact of Liver Ablative Radiotherapy using Kilovoltage intrafraction monitoring
}

Yoo Young Dominique Lee ${ }^{1,2^{*}}$ (D, Doan Trang Nguyen ${ }^{2,3,4}$, Trevor Moodie ${ }^{5}$, Ricky O'Brien ${ }^{4,6}$, Anne McMaster ${ }^{7}$, Andrew Hickey ${ }^{5}$, Nicole Pritchard ${ }^{5,8}$, Per Poulsen ${ }^{9}$, Elizaveta Mitkina Tabaksblat ${ }^{9}$, Britta Weber ${ }^{9}$, Esben Worm ${ }^{9}$, David Pryor ${ }^{1}$, Julie Chu ${ }^{10}$, Nicholas Hardcastle ${ }^{1}$, Jeremy Booth ${ }^{11}$, Val Gebski ${ }^{12}$, Tim Wang ${ }^{5}$ and Paul Keall ${ }^{4,6}$

\begin{abstract}
Background: Stereotactic Ablative Body Radiotherapy (SABR) is a non-invasive treatment which allows delivery of an ablative radiation dose with high accuracy and precision. SABR is an established treatment for both primary and secondary liver malignancies, and technological advances have improved its efficacy and safety. Respiratory motion management to reduce tumour motion and image guidance to achieve targeting accuracy are crucial elements of liver SABR. This phase II multi-institutional TROG 17.03 study, Liver Ablative Radiotherapy using Kilovoltage intrafraction monitoring (LARK), aims to investigate and assess the dosimetric impact of the KIM real-time image guidance technology. KIM utilises standard linear accelerator equipment and therefore has the potential to be a widely available real-time image guidance technology for liver SABR.

Methods: Forty-six patients with either hepatocellular carcinoma or oligometastatic disease to the liver suitable for and treated with SABR using Kilovoltage Intrafraction Monitoring (KIM) guidance will be included in the study. The dosimetric impact will be assessed by quantifying accumulated patient dose distribution with or without the KIM intervention. The patient treatment outcomes of local control, toxicity and quality of life will be measured.

Discussion: Liver SABR is a highly effective treatment, but precise dose delivery is challenging due to organ motion. Currently, there is a lack of widely available options for performing real-time tumour localisation to assist with accurate delivery of liver SABR. This study will provide an assessment of the impact of KIM as a potential solution for real-time image guidance in liver SABR.
\end{abstract}

(Continued on next page)

\footnotetext{
* Correspondence: Yooyoung.Lee@health.qld.gov.au

'Department of Radiation Oncology, Princess Alexandra Hospital, Brisbane,

QLD, Australia

${ }^{2}$ The University of Sydney, Sydney, NSW, Australia

Full list of author information is available at the end of the article
}

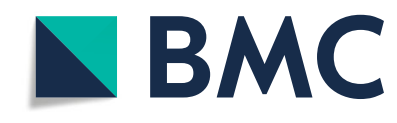

(c) The Author(s). 2021 Open Access This article is licensed under a Creative Commons Attribution 4.0 International License, which permits use, sharing, adaptation, distribution and reproduction in any medium or format, as long as you give appropriate credit to the original author(s) and the source, provide a link to the Creative Commons licence, and indicate if changes were made. The images or other third party material in this article are included in the article's Creative Commons licence, unless indicated otherwise in a credit line to the material. If material is not included in the article's Creative Commons licence and your intended use is not permitted by statutory regulation or exceeds the permitted use, you will need to obtain permission directly from the copyright holder. To view a copy of this licence, visit http://creativecommons.org/licenses/by/4.0/. The Creative Commons Public Domain Dedication waiver (http://creativecommons.org/publicdomain/zero/1.0/) applies to the data made available in this article, unless otherwise stated in a credit line to the data. 
(Continued from previous page)

Trial registration: This trial was registered on December 7th 2016 on ClinicalTrials.gov under the trial-ID NCT02 984566.

Keywords: Stereotactic radiotherapy, Liver Cancer, Hepatocellular carcinoma, Oligometastases, Kilovoltage intrafraction monitoring, LARK trial

\section{Background}

Stereotactic Ablative Body Radiation Therapy (SABR) also known as Stereotactic Body Radiation Therapy (SBRT) is a technique used to deliver high-precision, ablative doses of radiation in a small number of fractions to an extra-cranial target [1].

Worldwide, there has been a rapid adoption of SABR to treat a variety of malignancies [2] at a range of sites including lung [3], liver [4] and spine [5]. In the setting of the liver, SABR is an effective and a potentially curative treatment for hepatocellular carcinomas [6] and oligometastatic disease [7].

SABR requires accurate knowledge of the location of a tumour and its physiological motion in relation to surrounding structures. Intrafraction motion can result in geographical inaccuracies in SABR treatment and these, in turn, can compromise treatment outcome and have the potential to increase toxicity. A major challenge for liver SABR is the management of respiratory motion and multiple techniques have been introduced to either proactively manage respiratory motion (e.g. through active breathing control and respiratory gating) or reduce liver motion (e.g. abdominal compression). In liver SABR, implanting radio-opaque fiducial markers around a tumour may allow more accurate and precise localisation of the target volume during treatment delivery. Wahl et al., demonstrated $0 \%$ local failure in patients who received liver SABR with fiducial placement to guide treatment delivery [8]. Real-time tumour tracking, intuitively, would also elevate the confidence of precise dose delivery.

The current Image Guided Radiation Therapy (IGRT) standard of care requires a cone-beam computed tomography $(\mathrm{CBCT})$ scan prior to the beam delivery for localisation followed by appropriate adjustment of the treatment couch and verification $\mathrm{CBCT}$. This process can be repeated between the treatment beams and upon completion of the treatment to ensure accurate patient positioning during treatment. The potential benefits of intra-fraction monitoring are numerous. Without the continuous knowledge of the target position during the beam delivery, inaccurate dose delivery may result in suboptimal disease control and increased toxicities. Kilovoltage Infraction Monitoring (KIM) is an emerging real-time IGRT method. The ability for KIM to achieve real-time imaging to trigger manual pause of the beam delivery when the tumour motion exceeds a pre-set tolerance (Fig. 1) has been clinically investigated for prostate cancer SABR. A phase II study Stereotactic Prostate Adaptive Radiotherapy utilising Kilovoltage intrafraction monitoring (TROG SPARK 15.01) recently demonstrated the clinical benefit of intra-fraction monitoring with KIM guidance in prostate cancer [9]. In a recent computational study exploring the benefit of MLC tracking using KIM guidance in liver SABR, KIM detected much larger intrafraction monitoring than the pre-set tolerance [10], highlighting the importance of future studies in this space. Additionally, a separate study showed that with the application of gating or tracking, more patients are able to receive full isotoxic prescription [11]. The LARK trial will be the first clinical application of KIM outside of the prostate.

There are a range of technologies that provide real-time monitoring of the liver target. Direct image guidance without the need for markers can be achieved using ultrasound [12] or an MRI-Linac [13] 2D position monitoring for liver SABR achievable with markers using Elekta's XVI or Varian's Triggered Imaging. 3D position monitoring for liver SABR has been achieved with Calypso (Varian Medical Systems, Palo Alto, CA) [14] and a combined internalexternal monitoring system 'COSMIK' [15]. Both of the latter studies were from Aarhus University. Their institutional experience of liver SABR using Calypso continuous internal electromagnetic-based gating included 15 patients [14]. This study reported improved geometric and dosimetric accuracy compared with standard treatment. Potentially, KIM has a few practical benefits over the Calypso system. Firstly, KIM uses existing imaging equipment on a standard linear accelerator to achieve intrafraction motion monitoring. Secondly, when simulation MRIs are used for target volume delineation in liver SABR, the standard gold fiducials which are used for KIM are better visualised than the Calypso electromagnetic transponders. Their institutional experience of liver SABR using COSMIK combined $\mathrm{x}$-ray and respiratory signal method to achieve real-time guidance on a standard linear accelerator [16]. COSMIK has been prospectively implemented for liver SABR. With the combined use of the respiratory signal, COSMIK requires fewer $\mathrm{x}$-ray images than KIM and has a lower latency. On the other hand, KIM provides rotation in addition to translation and may have higher accuracy than COSMIK as it only uses the x-ray images. 


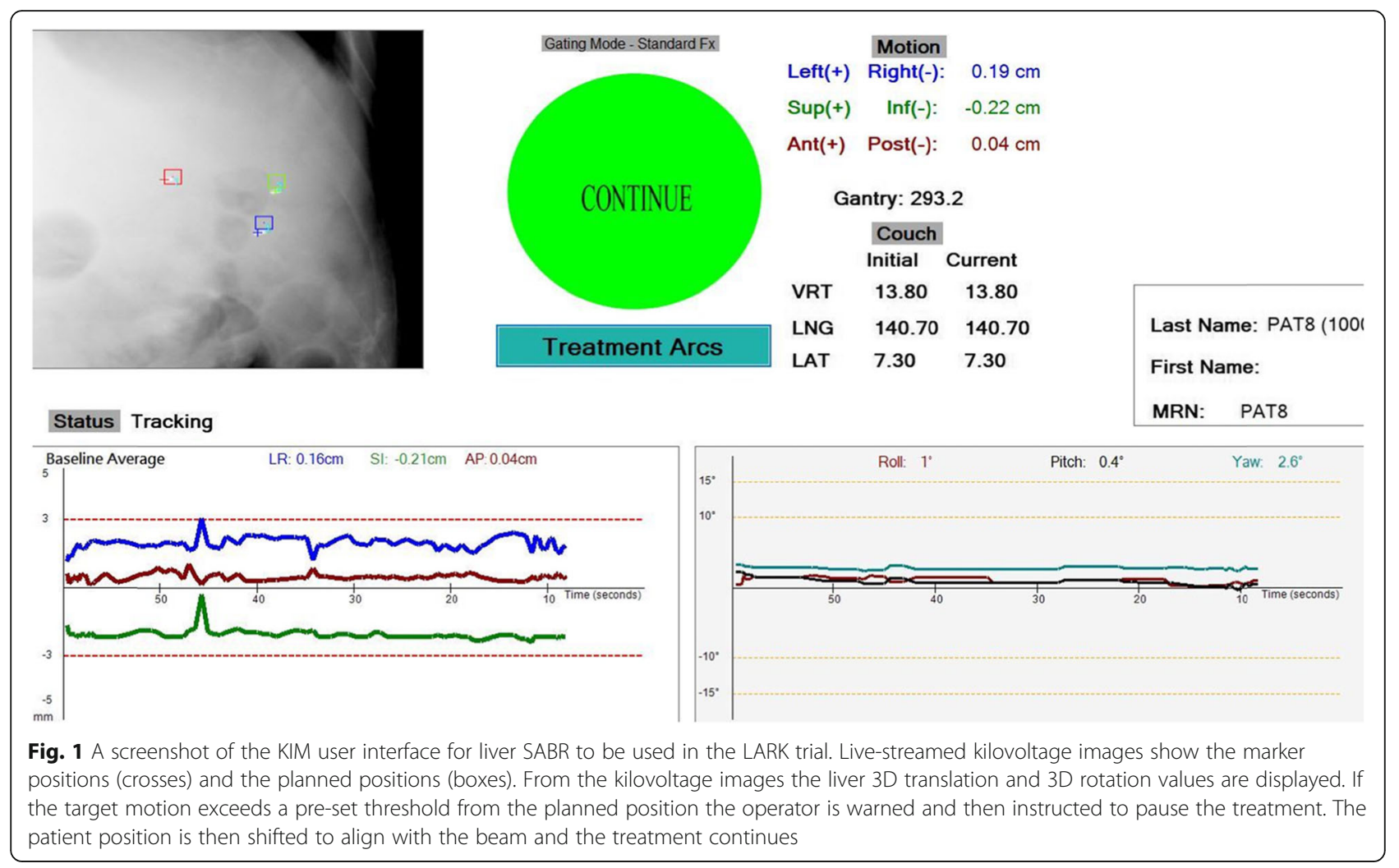

In addition to higher accuracy in treatment delivery, another potential benefit of using KIM is the reduction in imaging dose and faster treatment time. A retrospective analysis of patients treated with liver SABR at one of our institutions demonstrated an average of 5 CBCTs for each fraction resulting in 25 CBCTs per treatment course which translates to a total effective dose from imaging to be $186 \mathrm{mSv}$. The final effective dose from using an initial CBCT and KIM has been calculated to be 43 $\mathrm{mSv}$ which is a substantial reduction in imaging dose. In liver SABR, CBCT acquisition and analysis takes a significant portion of the treatment duration. Reducing the number of CBCTs could result in an important reduction of overall treatment time. A reduction in treatment time will increase patient throughput and also improve the patient experience, particularly for patients who experience pain in the supine position or are uncomfortable with their arms above their head for long periods of time.

An alternative method to KIM, COSMIK, also uses a combined $\mathrm{x}$-ray and respiratory signal method to achieve real-time guidance on a standard linear accelerator [16]. COSMIK has been prospectively implemented for liver SABR. With the combined use of the respiratory signal, COSMIK requires fewer $x$-ray images than KIM and has a lower latency. On the other hand, KIM provides rotation in addition to translation and may have higher accuracy than COSMIK as it only uses the $\mathrm{x}$-ray images.
The primary goal of the LARK trial is to assess the dosimetric impact of the KIM real-time IGRT technology, which utilises standard linear accelerator equipment, for liver cancer SABR.

\section{Methods/design}

This study is designed as a multi-institutional single arm phase II study. The LARK trial co-ordination will be conducted by the Trans Tasman Radiation Oncology Group (TROG). Forty-six patients who are eligible for liver SABR treatment with either primary or secondary liver malignancy will be treated with the incorporation of KIM. This study has been approved by Western Sydney Local Health District Research Ethics Board.

Each participating site will be required to undertake a credentialing procedure for review before enrolling any patients. The credentialing process includes the submission of a treatment plan meeting the planning criteria, the completion of the KIM commissioning and quality assurance procedures and an independent review. The KIM commissioning tests were adapted for liver cancer monitoring from $\mathrm{Ng}$ et al. [17]. The tests include static tests to ensure coincidence of coordinate systems between KIM and the linear accelerator, dynamic tests with liver patients' breath-hold and free breathing motion trajectories [15] and treatment interruption tests in which KIM is used to interrupt the treatment. For each test, KIM passes if the mean error of KIM reported 
results, as compared with the input motion, are less than $1 \mathrm{~mm}$ with a standard deviation of less than $2 \mathrm{~mm}$. Ongoing KIM quality assurance tests with the same criteria will be performed throughout the trial at a recommended interval of 1 month.

We aim to test the hypotheses that KIM improves cancer targeting accuracy, patient dose and outcomes. This improvement is defined as the success rate which is the ability of a KIM-corrected patient dose distribution to improve the Planning Target Voume (PTV) dose to 95\% of the volume $\left(D_{95}\right)$ or the liver dose to $50 \%$ of the volume $\left(D_{50}\right)$ by $5 \%$ compared to the patient dose distribution without KIM.

This study will accrue patients from five to seven institutions across Australia and one in Denmark. The dataset generated during and/or analysed during the current study are available from the corresponding author on reasonable request.

The study schema is shown in Fig. 2.

Following each treatment, dosimetric calculations will be performed to compare the dosimetric accuracy of the treatment with KIM incorporation against treatment that would have been delivered in the absence of KIM. Technological assessment of KIM to quantify the clinical practice impact as well as treatment outcome data will be obtained.

\section{Primary endpoint}

The primary outcome is to measure the dosimetric impact of the KIM real-time IGRT technology for liver cancer SABR. A dose accumulation method will be used to determine the dose delivered to the patient with KIM, and also the dose distributions that would have been delivered to the patient had KIM not been used. The isodose distributions and dose volume histograms for each session will be calculated and compared.

\section{Secondary endpoints}

- Assess treatment outcome

$\bigcirc$ Local control, toxicity and quality of life

- Quantitative assessment of treatment time, imaging dose, PTV margins, CBCT dose reconstruction

- Qualitative assessment of dose reduction with MLC tracking (simulation study only)

\section{Key selection criteria}

All patients eligible for liver SABR (with fiducial marker placement) for hepatocellular carcinoma or secondary liver malignancy are eligible for this study.

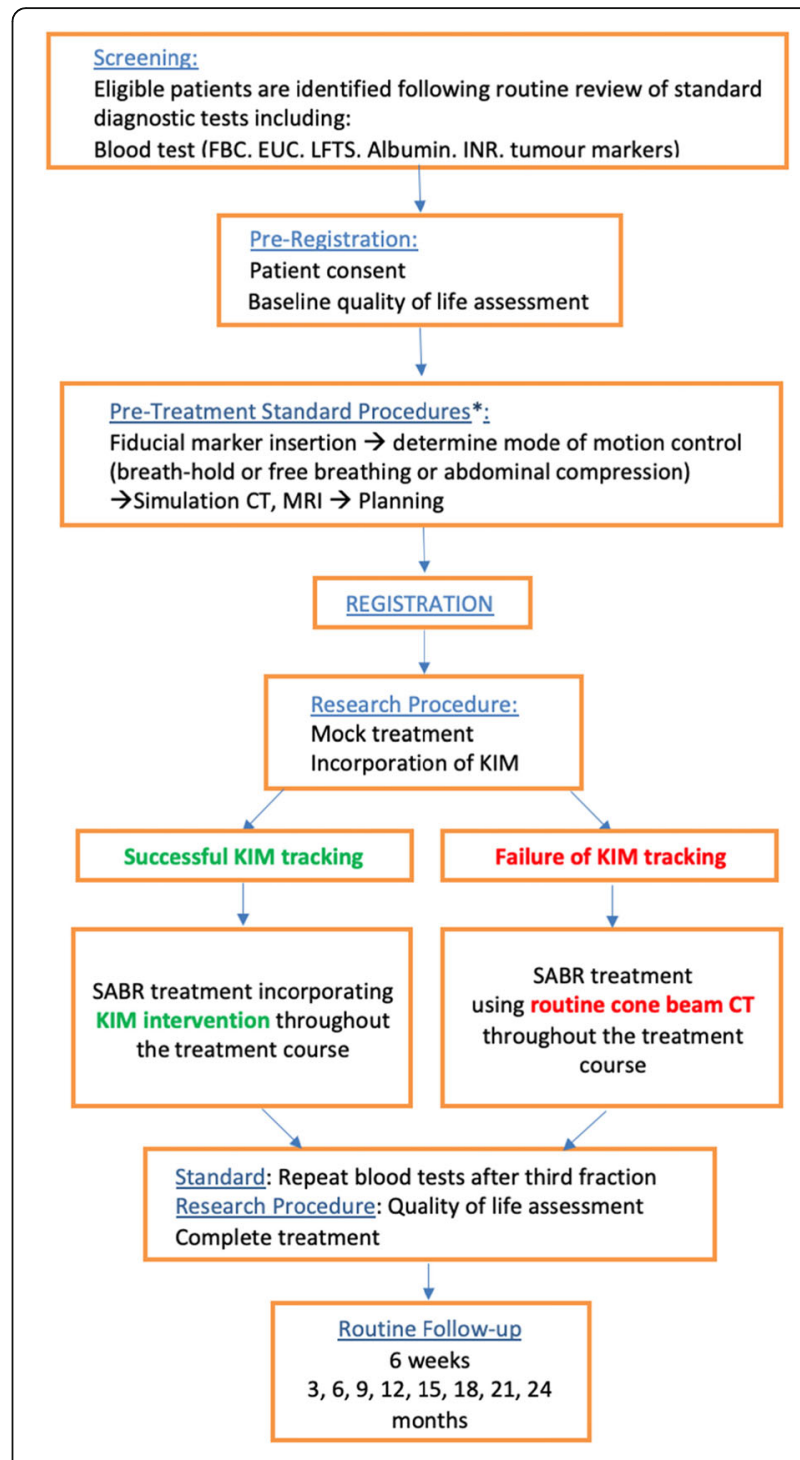

Fig. 2 The LARK study schema

Inclusion

HCC

- Diagnosed by either radiological guidelines $(>1 \mathrm{~cm}$, enhancing arterial phase and wash-out in later phase) or biopsy

- Child-Pugh stage A/B7

\section{Liver metastases}

- Biopsy preferred but detected on imaging allowed

- Controlled primary tumour: at least 3 months since original tumour treated with curative intent, with no progression at primary site

Patients will be required to be age 18 or over, ECOG performance status $0-2$ with life expectancy greater than 
6 months. Three or fewer liver lesions, lesion size less than $10 \mathrm{~cm}$ in the largest diameter in any direction for a single lesion (and up to $10 \mathrm{~cm}$ cumulative diameter for multiple lesions), unsuitable for RFA/MWA. Patients may have had previous surgery, RFA/MWA or ethanol injection, or TACE. All blood work obtained within 6 weeks prior to study entry with adequate organ function. Patients must have been discussed at a multidisciplinary tumour board with the consensus opinion for SABR.

\section{Exclusion}

Patients with hepatocellular carcinoma who have evidence of metastatic disease including nodal or distant metastases, patients with metastatic disease who have had complete liver disease response to first-line chemotherapy (i.e. no target for SABR). Previous radiation to the liver (including SIRTEX), untreated HIV or active hepatitis B, systemic antineoplastic drug therapy within 7 days before inclusion, pregnant or lactating women.

Patients with less than two fiducial markers and/or fiducial markers with greater than $10 \mathrm{~cm}$ will be excluded.

\section{Radiotherapy planning and treatment Pre-treatment}

Fiducial markers are mandatory for this study. The recommended number of fiducial markers is three or more to allow triangulation and measurement of position in different planes. Fiducials should be inserted $1-2 \mathrm{~cm}$ from the tumour to be viable surrogates. To serve a purpose as a better surrogate than using anatomical landmarks, Seppenwoolde et al., recommended the placement of the makers closer than $8 \mathrm{~cm}$ to the tumour centre [18]. Fiducial markers should be implanted at least 1 day prior to simulation.

\section{Simulation}

Prior to CT simulation, it is recommended that patients undergo a motion management assessment such as fluoroscopy to determine the best choice of motion management. Assessment could include end-expiration breath-hold (EEBH), deep inspiration breath-hold $(\mathrm{DIBH})$, and free-breathing (FB) with or without abdominal compression. EEBH is the preferred motion management method.

A triple phase CT with contrast and a non-contrast CT should be obtained using maximum $3 \mathrm{~mm}$ thickness slices (1 mm preferred). For patients who are suitable for EEBH or DIBH, all scans should be done at breath-hold. A 4DCT should be acquired if free-breathing with or without abdominal compression is utilised to determine tumour motion.

Contrast enhanced MRI can be used to tumour delineation preferably in treatment position on a flat couch top utilising the same motion management method as for the planning CTs. PET-CT may be used for patients with liver metastases.

\section{Treatment planning \\ Target volumes}

Using non-contrast CT as the primary dataset, the target volumes are defined as:

- Gross Tumour Volume (GTV) = tumour visible on CT and/or MRI, ideally after review with diagnostic radiologist and consideration of other imaging available (e.g. PET scan for liver metastases).

- Clinical Target Volume (CTV) = GTV

- Internal Target Volume (ITV) is optional but should be established if the patient is to be treated with free breathing or abdominal compression techniques. The magnitude of motion may be determined by measuring the trajectory of the fiducial markers or other surrogate on a 4DCT scan.

- Planning Target volume (PTV) $=\mathrm{CTV}+5-15 \mathrm{~mm}$ depending on motion management strategy. For treatment using EEBH or DIBH, typically $5 \mathrm{~mm}$ in radial directions, and $5-7 \mathrm{~mm}$ in cranio-caudal directions from the CTV. Note that the margin can be different in all planes. For treatment using freebreathing, typically $5 \mathrm{~mm}$ in all direction from the ITV.

\section{Dose prescription}

The range of dose prescriptions and fractionation schedules including the planning dose constraints are adapted from existing recommendations [19-21] and clinical trials including UK CORE, RTOG 1112, NRG BR001, agreed upon by radiation oncologists from six institutions across Australia and Denmark with expertise in liver SABR.

\section{Hepatocellular carcinoma}

Six dose prescriptions (50 Gy, 45 Gy, 40 Gy, 35 Gy, 30 Gy or $27.5 \mathrm{~Gy}$ ) in five fractions depending on the clinical scenario and the radiation oncologist choice. Interfraction time should be between 24 and $72 \mathrm{~h}$ and treatment should be delivered over 5-15 days.

\section{Liver metastases}

Two fractionation regimens are available depending on the clinical scenario and the radiation oncologist choice. Generally, three fractions are preferred. For three fraction regimens, the dose prescription levels include 54Gy, $51 \mathrm{~Gy}, 48 \mathrm{~Gy}, 45 \mathrm{~Gy}$ or $42 \mathrm{~Gy}$ delivered on alternate days over 5-7 days. For five fraction regimens, the dose prescription levels are 60 Gy, 55 Gy, 50 Gy, 45 Gy 40 Gy delivered over 10-15 days with a preferred interfraction interval greater than $36 \mathrm{~h}$. 


\section{Treatment techniques}

Volumetric Modulated Arc Radiotherapy (VMAT) or IMRT (Intensity Modulated Radiation Therapy) planning is required and flattening filter free beams are allowed. A real-time pre-treatment quality assurance technical review will be performed via TROG for all participants. Target volume planning goals outlined in Table 1 and organ at risk planning guidelines are provided in the LARK Radiation Therapy Quality Assurance document. The planning dose-volume constraints (supplementary Tables 1-4 are appended to this manuscript).

\section{Treatment delivery}

Each participant will undergo a pre-treatment assessment session prior to the first fraction to assess feasibility of KIM. During this pre-treatment session, a CBCT scan will be acquired. KIM will be used to track the fiducials on the projection images during at least $2 \mathrm{CBCT}$ acquisitions. KIM reported 3D positions will be compared with manual fiducial matching by the radiation therapists on the reconstructed CBCT. If the KIM reported couch shift is $2 \mathrm{~mm}$ or less from the manual match couch shift, the treatment course will commence with KIM. In scenarios where KIM fails to track the fiducial markers, or the KIM reported couch shift is more than $2 \mathrm{~mm}$ from the manual match the current standard-or-care IGRT with CBCT will be utilised for the patient treatment.

During patient treatments, following the initial CBCT and patient repositioning, KIM will be used to provide continuous monitoring of the tumour motion during treatment beam-on. If using breath-hold technique, fiducial displacement of $3 \mathrm{~mm}$ from the baseline for more than $5 \mathrm{~s}$, the beam will be manually turned off. If the fiducial does not return to baseline for two consecutive breath-holds, the patient will be realigned by shifting the couch. For free-breathing or abdominal compression technique, if the fiducials exceed the ITV-based threshold for more $5 \mathrm{~s}$ the beam will be turned off until the fiducial markers return within tolerance. If the fiducials exhibit a baseline shift, the patient will be realigned with a couch shift.

For both breath-hold and free-breathing treatments, participating institutions are permitted and encouraged to use their clinical standard motion management techniques including optical devices such as Real-time Position Management (RPM, Varian, Palo Alto, CA, USA), Active Breathing Coordinator (ABC, Elekta, Stockholm, Sweden) or AlignRT (VisionRT, London, UK).

\section{Assessments}

Dosimetric / technological assessment For each fraction, the targeting accuracy and delivered patient dose distribution will be determined via paired control by comparing the measured targeting error and dose with KIM to those that would have been delivered in the absence of KIM.

We will also perform a technology assessment of KIM to quantify the clinical practice impact by:

1. Quantifying the impact on workflow using KIM through time-motion studies

2. Evaluating operator and clinician confidence in KIM's reliability and clinical efficacy through a technology-impact survey

3. Quantifying the system robustness through hardware and software fault reporting

4. Performing system quality assurance (QA), at multiple sites, sharing the results through a webbased upload and provide feedback for QA improvement

Acute / late toxicity and disease outcome assessment Patients will undergo clinical assessment as well as

Table 1 Target volume doses for the TROG 17.03 LARK clinical trial. TD = Target Dose (prescribed dose). RVR= Remaining Volume at Risk

\begin{tabular}{|c|c|c|c|c|c|c|c|}
\hline \multicolumn{8}{|c|}{ LARK Target Volume Doses } \\
\hline \multirow{2}{*}{$\begin{array}{l}\text { Standardised } \\
\text { Name }\end{array}$} & \multirow[t]{2}{*}{ Constraint } & \multicolumn{2}{|c|}{ Per Protocol } & \multicolumn{2}{|l|}{ Minor Variation } & \multicolumn{2}{|l|}{ Major Variation } \\
\hline & & $\begin{array}{l}\text { PTVs } \\
\text { around } \\
\text { GTVs }\end{array}$ & $\begin{array}{l}\text { PTVs around } \\
\text { Non-GTV } \\
\text { CTVs }^{\mathrm{a}} \\
\end{array}$ & PTVs around GTVs & $\begin{array}{l}\text { PTVs around Non- } \\
\text { GTV CTVs }^{\mathrm{a}}\end{array}$ & $\begin{array}{l}\text { PTVs around } \\
\text { GTVs }\end{array}$ & $\begin{array}{l}\text { PTVs around } \\
\text { Non-GTV CTVs }{ }^{a}\end{array}$ \\
\hline GTV & D100\% & $\geq 100 \%$ o & the TD & $95-100 \%$ of the TD & & $\leq 95 \%$ of the TD & \\
\hline \multirow[t]{2}{*}{ PTV } & $\begin{array}{l}\text { Dmax } \\
(0.03 \mathrm{cc})\end{array}$ & \multicolumn{2}{|c|}{$120-140 \%$ of the TD } & $140-150 \%$ of the TD or & $110-120 \%$ of the TD & \multicolumn{2}{|c|}{$\geq 150 \%$ of the TD or $\leq 110 \%$ of the TD } \\
\hline & D95\% & \multicolumn{2}{|c|}{$95-105 \%$ of the TD } & $\begin{array}{l}90-95 \% \text { or } 105-110 \% \\
\text { of the TD, and } \geq 25 \mathrm{~Gy}\end{array}$ & $\begin{array}{l}85-95 \% \text { or } 105-115 \% \\
\text { of the TD, and } \geq 25 \mathrm{~Gy}\end{array}$ & $\begin{array}{l}\leq 90 \% \text { or } \geq 110 \% \text { of } \\
\text { the TD, or } \leq 25 \mathrm{~Gy}\end{array}$ & $\begin{array}{l}\leq 85 \% \text { or } \geq 115 \% \text { of } \\
\text { the } \mathrm{TD} \text {, or } \leq 25 \mathrm{~Gy}\end{array}$ \\
\hline RVR & $\begin{array}{l}\text { Dmax } \\
(0.03 \mathrm{cc})\end{array}$ & \multicolumn{2}{|c|}{$\leq 120 \%$ of the TD } & - & & \multicolumn{2}{|l|}{$\geq 120 \%$ of the TD } \\
\hline
\end{tabular}

a Non-GTV CTVs represents regions at high risk for microscopic disease, including non-tumour vascular thrombi, prior TACE sites, or adjacent RFA or other ablation sites. Treatment of these high-risk sites are permitted 
routine blood tests, imaging at pre-defined time points as outlined in Fig. 2. Both acute and late toxicity will be reported using NCI Common Terminology Criteria for Adverse Events (CTCAE) version 4 and Radiation Induced Liver Disease (RILD).

Treatment response will be assessed by triple-phase CT or MRIs for patients with HCC. For patients with metastatic liver disease, local control will be assessed with CT scan and /or PET CT or MRI. The preferred assessment for HCC is the modified RECIST criteria [22] and for metastatic disease RECIST and/or PERCIST criteria [23].

Patient-reported outcomes (PROs) will be assessed using the general EORTC QLQ-C30 and disease specific quality of life questionnaires EORTC QLQ-HCC18.

In this study, 46 patients are needed to test the hypothesis that KIM can improve patient dose distribution.

We consider a treatment a success if the KIMcorrected patient dose distribution improves the planning target volume (PTV) dose to $95 \%$ of the volume $\left(D_{95}\right)$ or the liver dose to $50 \%$ of the volume $\left(D_{50}\right)$ by $5 \%$ compared to dosimetry without KIM. A dose accumulation method [24] will be used to determine the efficacy of KIM, where the isodose distributions and dose volume histograms for each treatment course will be calculated with KIM corrections as treated, and estimated without KIM corrections.

A $60 \%$ or higher treatment success rate would suggest KIM is promising and worthy of further investigation. Alternatively, a treatment success rate of $40 \%$ or lower would suggest than KIM should not be considered for this treatment modality. Using Simon's two-stage optimum design [25], a sample size of 46 patients will have $80 \%$ power with $95 \%$ confidence to rule out a $40 \%$ success rate in favour of a more interesting success rate of $60 \%$.

Interim analysis for futility The success rate will be determined after 16 patients have been accrued and completed treatment. If fewer than nine successes are observed, consideration will be given to re-examining the cause of the lack of success and the trial could be redesigned or stopped for futility at this point. However, recruitment will continue while this futility boundary is evaluated. If nine or more success are observed, 30 additional patients will be accrued for a total of 46 patients. The main analysis will be performed after at least $46 \mathrm{pa}-$ tients have been followed for 2 years, or the data is sufficiently mature to report the results earlier.

Exploratory analyses comparing outcomes of the KIM 'failures' to KIM 'successes' will also be performed to obtain estimates of whether the degree of accuracy afforded by KIM actually translates to clinical endpoints.

\section{Discussion}

This multi-centre study aims to accrue 46 patients to test the hypothesis that KIM provides a real-time IGRT solution to improve cancer targeting accuracy, dose delivery and treatment outcomes in patients receiving liver SABR. Several studies have demonstrated liver motion up to several centimetres during treatment $[15,26,27]$ and a retrospective review at our institution also demonstrated significant intrafraction motion which would not have been observed without using a real-time imaging system such as KIM.

Other potential benefits of KIM technology include a reduction in imaging radiation dose and overall treatment time compared to current IGRT method of using CBCT. We have demonstrated that continuous kilovoltage imaging results in substantially less imaging dose than multiple CBCT scans. CBCTs are acquired during breath-holds, which is often the reason for long treatment times leading to patient fatigue and instability in the patients' ability to maintain consistent breath-holds.

The liver is a radiosensitive organ, and particularly in patients with liver cirrhosis, maximum sparing of the functioning liver is a critical goal in SABR. Continuous intrafraction monitoring has the potential to improve the accuracy of treatment delivery for better disease outcome and reduced treatment-related toxicity. Ultimately, if direct intra-fraction visualisation of the target lesion using KIM method is successful, it may help to safely reduce the PTV margins to maximise further liver sparing and minimise toxicity.

\begin{abstract}
Abbreviations
CBCT: Cone-Beam Computed Tomography; CTV: Clinical Tumour Volume; DIBH: Deep Inspiration Breath-Hold; EEBH: End-Exhale Breath-Hold; GTV: Gross Tumour Volume; IGRT: Image Guided Radiation Therapy; IMRT: Intensity Modulated Radiotherapy; ITV: Internal Target Volume; KIM: Kilovoltage Intrafraction Monitoring; LARK: Liver Ablative Radiotherapy using Kilovoltage intrafraction monitoring; MLC: Multileaf Collimator; PERC IST: Positron Emission Tomography Response Criteria In Solid Tumours; PTV: Planning Target Volume; QA: Quality Assurance; RECIST: Response Evaluation Criteria In Solid Tumours; RILD: Radiation Induced Liver Disease; SABR: Stereotactic Ablative Body Radiotherapy; SBRT: Stereotactic Body Radiation Therapy; VMAT: Volumetric Modulated Arc Therapy
\end{abstract}

\section{Supplementary Information}

The online version contains supplementary material available at https://doi. org/10.1186/s12885-021-08184-x

\section{Additional file 1.}

\section{Acknowledgements}

We would like to thank the Trans Tasman Radiation Oncology Group (TROG) and University of Sydney for supporting this trial.

Authors' contributions

Funding application: PK. Principle Investigators: YL and TW. Conceptual Design and Development: YL, TW, DTN, TM, AM, PK. Drafting of the Protocol Manuscript: YL, DTN, TW, PK. Intellectual Content: All authors, Study supervision: TW, PK, Revision and Final Approval of the Article: All authors. 


\section{Funding}

This trial is funded by the Australian Government Cancer Australia awarded to PK following a peer-review process. The funding body has no role in collecting, analysing or interpreting of the trial data.

\section{Availability of data and materials}

The LARK Trial Management Committee support the use of the data acquired in this study for further research within the bounds of patient privacy and ethics concerns. The protocol states: De-identified data collected in this study will be stored perpetually and may be used for future research. The stored data will only be used in future research under the approval of a Human Research Ethics Committee. Researchers interested in the use of the LARK trial data should contact the study authors.

\section{Declarations}

\section{Ethics approval and consent to participate}

This study (NCT02984566) has received ethical approval from Western Sydney Local Health District Research Ethics Board on the 24th of October 2016. Written informed consent is obtained from all participants before inclusion.

This protocol manuscript is in accordance with SPRIT 2013 recommendations and the corresponding author has completed the checklist.

\section{Consent for publication}

Not applicable.

\section{Competing interests}

PK is an inventor on an issued patent related to the KIM technology that is licensed from Stanford University to Varian Medical Systems, and an inventor and owner on other filed patents related to the KIM technology that are unlicensed.

\section{Author details}

${ }^{1}$ Department of Radiation Oncology, Princess Alexandra Hospital, Brisbane, QLD, Australia. ${ }^{2}$ The University of Sydney, Sydney, NSW, Australia. ${ }^{3}$ School of Biomedical Engineering, University of Technology Sydney, Sydney, NSW, Australia. ${ }^{4}$ ACRF Image X Institute, Sydney, NSW, Australia. ${ }^{5}$ Department of Radiation Oncology, Crown Princess Mary Cancer Centre, Sydney, NSW, Australia. ${ }^{6}$ Radiation Physics Laboratory, Sydney Medical School, The University of Sydney, Sydney, NSW, Australia. ${ }^{7}$ Department of Radiation Oncology, Liverpool-Macarthur Cancer Therapy Centre, Sydney, NSW Australia. ${ }^{8}$ Gamma Gurus Pty Ltd, Sydney, NSW, Australia. ${ }^{9}$ Department of Oncology, Aarhus University Hospital, Aarhus, Denmark. ${ }^{10}$ Department of Radiation Oncology, Peter MacCallum Cancer Centre, Melbourne, Victoria, Australia. ${ }^{11}$ Department of Radiation Oncology, Northern Sydney Cancer Centre, Sydney, NSW, Australia. ${ }^{12}$ University of Sydney NHMRC Clinical Trials Centre, Sydney, NSW, Australia.

Received: 24 November 2020 Accepted: 13 April 2021

Published online: 03 May 2021

\section{References}

1. Chang BK, Timmerman RD. Stereotactic body radiation therapy: a comprehensive review. Am J Clin Oncol. 2007;30(6):637-44. https://doi.org/1 0.1097/COC.0b013e3180ca7cb1

2. Lewis SL, Porceddu S, Nakamura N, Palma DA, Lo SS, Hoskin P, et al. Definitive stereotactic body radiotherapy (SBRT) for extracranial oligometastases: an international survey of $>1000$ radiation oncologists. Am J Clin Oncol. 2017;40(4):418-22. https://doi.org/10.1097/COC. 0000000000000169.

3. Timmerman R, Paulus R, Galvin J, Michalski J, Straube W, Bradley J, et al. Stereotactic body radiation therapy for inoperable early stage lung cancer. JAMA. 2010;303(11):1070-6. https://doi.org/10.1001/jama.2010.261.

4. Hoyer M, Swaminath A, Bydder S, Lock M, Mendez Romero A, Kavanagh B, et al. Radiotherapy for liver metastases: a review of evidence. Int J Radiat Oncol Biol Phys. 2012;82(3):1047-57. https://doi.org/10.1016/j.ijrobp.2011.07. 020.

5. Hall WA, Stapleford L, Hadjipanayis CG, Curran WJ, Crocker I, Shu HK. Stereotactic body radiosurgery for spinal metastatic disease: an evidencebased review. Int J Surg Oncol. 2011;2011:979214.
6. Murray $L$, Dawson LA. Advances in stereotactic body radiation therapy for hepatocellular carcinoma. Semin Radiat Oncol. 2017;27(3):247-55. https:// doi.org/10.1016/j.semradonc.2017.02.002.

7. Hellman S, Weichselbaum RR. Oligometastases. J Clin Oncol. 1995;13(1):810. https://doi.org/10.1200/JCO.1995.13.1.8.

8. Wahl DR, Stenmark MH, Tao Y, Pollom EL, Caoili EM, Lawrence TS, et al. Outcomes after stereotactic body radiotherapy or radiofrequency ablation for hepatocellular carcinoma. J Clin Oncol. 2016;34(5):452-9. https://doi. org/10.1200/JCO.2015.61.4925.

9. Keall P, Nguyen DT, O'Brien R, Hewson E, Ball H, Poulsen P, et al. Real-time image-guided ablative prostate cancer radiation therapy: results from the TROG 15.01 spark trial. Int J Radiat Oncol Biol Phys. 2020;107:530-8.

10. Poulsen PR, Murtaza G, Worm ES, Ravkilde T, O'Brien R, Grau C, et al. Simulated multileaf collimator tracking for stereotactic liver radiotherapy guided by kilovoltage intrafraction monitoring: Dosimetric gain and target overdose trends. Radiother Oncol. 2020;144:93-100. https://doi.org/10.1016/ j.radonc.2019.11.008

11. Gargett M, Haddad C, Kneebone A, Booth JT, Hardcastle N. Clinical impact of removing respiratory motion during liver SABR. Radiat Oncol. 2019;14(1): 93. https://doi.org/10.1186/s13014-019-1300-6.

12. Boda-Heggemann J, Dinter D, Weiss C, Frauenfeld A, Siebenlist $K$, Attenberger $U$, et al. Hypofractionated image-guided breath-hold SABR (stereotactic ablative body radiotherapy) of liver metastases--clinical results. Radiat Oncol. 2012;7(1):92. https://doi.org/10.1186/1748-717X-7-92.

13. Witt JS, Rosenberg SA, Bassetti MF. MRI-guided adaptive radiotherapy for liver tumours: visualising the future. Lancet Oncol. 2020;21(2):e74-82. https://doi.org/10.1016/S1470-2045(20)30034-6.

14. Worm ES, Hoyer M, Hansen R, Larsen LP, Weber B, Grau C, et al. A prospective cohort study of gated stereotactic liver radiation therapy using continuous internal electromagnetic motion monitoring. Int J Radiat Oncol Biol Phys. 2018;101(2):366-75. https://doi.org/10.1016/j.jjrobp.2018.02.010.

15. Bertholet J, Worm ES, Fledelius W, Hoyer M, Poulsen PR. Time-resolved Intrafraction target translations and rotations during stereotactic liver radiation therapy: implications for marker-based localization accuracy. Int J Radiat Oncol Biol Phys. 2016;95(2):802-9. https://doi.org/10.1016/j.jjrobp.201 6.01.033.

16. Bertholet J, Toftegaard J, Hansen R, Worm ES, Wan H, Parikh PJ, et al. Automatic online and real-time tumour motion monitoring during stereotactic liver treatments on a conventional linac by combined optical and sparse monoscopic imaging with kilovoltage $x$-rays (COSMIK). Phys Med Biol. 2018;63(5):055012. https://doi.org/10.1088/1361-6560/aaae8b.

17. Ng JA, Booth JT, O'Brien RT, Colvill E, Huang CY, Poulsen PR, et al. Quality assurance for the clinical implementation of kilovoltage intrafraction monitoring for prostate cancer VMAT. Med Phys. 2014;41(11):111712. https://doi.org/10.1118/1.4898119.

18. Seppenwoolde Y, Wunderink W, Wunderink-van Veen SR, Storchi P, Mendez Romero A, Heijmen BJ. Treatment precision of image-guided liver SBRT using implanted fiducial markers depends on marker-tumour distance. Phys Med Biol. 2011:56(17):5445-68. https://doi.org/10.1088/0031-9155/56/17/001.

19. Benedict SH, Yenice KM, Followill D, Galvin JM, Hinson W, Kavanagh B, et al. Stereotactic body radiation therapy: the report of AAPM task group 101. Med Phys. 2010;37(8):4078-101. https://doi.org/10.1118/1.3438081.

20. Timmerman RD. An overview of hypofractionation and introduction to this issue of seminars in radiation oncology. Semin Radiat Oncol. 2008;18(4):21522. https://doi.org/10.1016/j.semradonc.2008.04.001.

21. Osmundson EC, Wu Y, Luxton G, Bazan JG, Koong AC, Chang DT. Predictors of toxicity associated with stereotactic body radiation therapy to the central hepatobiliary tract. Int J Radiat Oncol Biol Phys. 2015;91(5):986-94. https:// doi.org/10.1016/j.ijrobp.2014.11.028.

22. Oldrini G, Huertas A, Renard-Oldrini S, Taste-George H, Vogin G, Laurent V, et al. Tumor response assessment by MRI following stereotactic body radiation therapy for hepatocellular carcinoma. PLoS One. 2017;12(4): e0176118. https://doi.org/10.1371/journal.pone.0176118.

23. Pinker K, Riedl C, Weber WA. Evaluating tumor response with FDG PET: updates on PERCIST, comparison with EORTC criteria and clues to future developments. Eur J Nucl Med Mol Imaging. 2017;44(Suppl 1):55-66. https:// doi.org/10.1007/s00259-017-3687-3.

24. Poulsen PR, Schmidt ML, Keall P, Worm ES, Fledelius W, Hoffmann L. A method of dose reconstruction for moving targets compatible with dynamic treatments. Med Phys. 2012;39(10):6237-46. https://doi.org/10.111 8/1.4754297. 
25. Simon R. Optimal two-stage designs for phase II clinical trials. Control Clin Trials. 1989;10(1):1-10. https://doi.org/10.1016/0197-2456(89)90015-9.

26. Poulsen PR, Worm ES, Petersen JB, Grau C, Fledelius W, Hoyer M. Kilovoltage intrafraction motion monitoring and target dose reconstruction for stereotactic volumetric modulated arc therapy of tumors in the liver. Radiother Oncol. 2014;111(3):424-30. https://doi.org/10.1016/j.radonc.2014. 05.007.

27. Worm ES, Hoyer M, Fledelius W, Poulsen PR. Three-dimensional, timeresolved, intrafraction motion monitoring throughout stereotactic liver radiation therapy on a conventional linear accelerator. Int J Radiat Onco Biol Phys. 2013;86(1):190-7. https://doi.org/10.1016/j.jirobp.2012.12.017.

\section{Publisher's Note}

Springer Nature remains neutral with regard to jurisdictional claims in published maps and institutional affiliations.

Ready to submit your research? Choose BMC and benefit from:

- fast, convenient online submission

- thorough peer review by experienced researchers in your field

- rapid publication on acceptance

- support for research data, including large and complex data types

- gold Open Access which fosters wider collaboration and increased citations

- maximum visibility for your research: over $100 \mathrm{M}$ website views per year

At $\mathrm{BMC}$, research is always in progress.

Learn more biomedcentral.com/submissions 